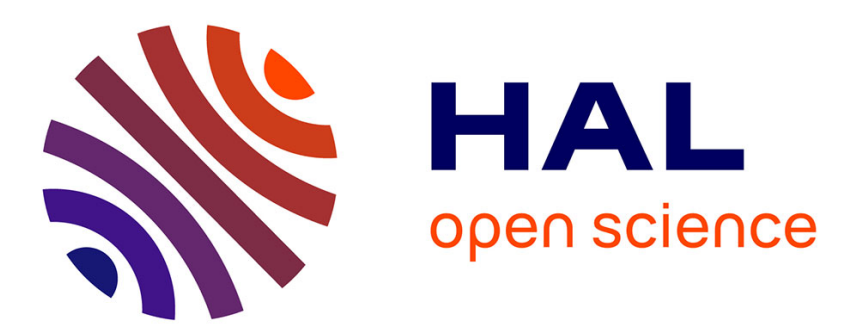

\title{
Improving rotation behaviour of robotic structures for micro-assembly.
}

David Hériban, Arnaud Thiebault, Michaël Gauthier, Guillaume Fortier

\section{To cite this version:}

David Hériban, Arnaud Thiebault, Michaël Gauthier, Guillaume Fortier. Improving rotation behaviour of robotic structures for micro-assembly.. 4th IEEE Internationale Conference on Automation Science and Engineering, IEEE CASE'08., Aug 2008, Key Bridge Marriott, Washington DC, United States. pp.983-988, 10.1109/COASE.2008.4626490 . hal-00323641

\section{HAL Id: hal-00323641 \\ https://hal.science/hal-00323641}

Submitted on 22 Sep 2008

HAL is a multi-disciplinary open access archive for the deposit and dissemination of scientific research documents, whether they are published or not. The documents may come from teaching and research institutions in France or abroad, or from public or private research centers.
L'archive ouverte pluridisciplinaire HAL, est destinée au dépôt et à la diffusion de documents scientifiques de niveau recherche, publiés ou non, émanant des établissements d'enseignement et de recherche français ou étrangers, des laboratoires publics ou privés. 


\title{
Improving rotation behaviour of robotic structures for micro-assembly
}

\author{
David Hériban, Arnaud Thiebault, Michaël Gauthier IEEE Member, Guillaume Fortier \\ FEMTO-ST Institute, UMR CNRS 6174 - UFC / ENSMM / UTBM \\ Automatic Control and Micro-Mechatronic Systems Department \\ 24, rue Alain Savary, 25000 Besançon, FRANCE \\ david.heriban at ens2m.fr, michael.gauthier at ens2m.fr
}

\begin{abstract}
Serial micro-assembly requires high precision robots able to produce translations and rotations to position and orient objects during assembly. In micro-scale, the translation ranges required are typically up to the millimeter and can be obtained with smart devices (piezomotor, etc.). In the other hand, the rotation ranges stay identical to the macroscale (eg. $90^{\circ}$ ) and require standard guidings like ball bearings which induce disturbances on the linear position. Thus the ability to produce high precision robots where translations and rotations are decoupled is currently one of the major stake in microassembly. This paper deals with an original modeling of the coupling between rotation and linear position. The geometrical model is presented and two calibration methods are discussed. Our method were tested on a 3 DOF planar robotic systems and the coupling was reduced by $93 \%$.
\end{abstract}

\section{INTRODUCTION}

Serial micro-assembly is an innovative way to perform out-of-plane and/or hybrid microsystems and requires a lot of innovative breakthrough. Three major domains are studied to improve micro-assembly: the study of new handling strategies adapted to the specificities of the micro-objects [1], [2]; the study of sensors able to measure position of the micro-object (eg. microvision) [3], and handling microforces [4]; the study of high precision robots able to position micro-objects with sufficient accuracy [5].

This paper focuses on this last problematics. To perform tridimensional assembly, robotic devices require degree of freedom in translation and rotation. As the objects manipulated are typically up to one millimeter, great accuracy, linearity and repeatability are excepted.

The translation range required in assembly is typically proportional to the size of the objects manipulated. Thus linear displacements and accuracy of the robot are reduced by scale-effect. In fact, typical ranges in micro-assembly are about hundreds micrometers to several millimeters. In this domain, several technologies have been explored like smart material actuation (piezoelectric structures, SMA, MSMA, etc.). Some commercial stages exist and provide linear displacement with a centimetric range and a repeatability around few micrometers (eg. PI Gmbh or MICOS).

Concerning the rotations, whatever the scale, tridimensional assembly requires always large rotations (eg. $180^{\circ}$ for

This work is supported by the french research project PRONOMIA ANR $n^{\circ}$ 05-BLAN-0325-01 an half turn). In other terms, angles are not modified by scale effect and rotation stages require the same range and the same angular accuracy (eg. $0.1^{\circ}$ ) for all scales. Smart materials are only able to induce small displacements (in translation and in rotation too) and thus can not be used. Rotations are currently done with standard actuation principle, and standard guidings like ball bearings. The accuracy in rotation obtained is sufficient (typically better than $0.1^{\circ}$ ).

However, coupling between rotation and position has to be considered too. In one hand the impact of linear stages on rotation (pitch, roll and yaw disturbing motion) could be first neglected because of the quite large angular repeatability expected. In the other hand, the impact of rotation stages on linear displacements (eccentricity and wobble) is usually higher than the repeatability expected in translation (typ. down to one micrometer). In fact, standard actuation principle used in rotation do not guarantee a constant position of the center of rotation. This center is a function of the angular position of the stage, and consequently angular motion highly perturbs linear position. Consequently, the translation repeatability of the whole robotic system stays coarse despite the use of smart linear actuators. The reduction of the coupling between rotation and translation in microrobotics is thus currently one of the major stakes in the automation of robotic micro-assembly.

Current works on robotic calibration for micro-assembly are focused on the modeling of 2D or 3D linear stages or on rotation stages obtained by deformation which can not create large rotations [6], [7], [8].

The objective of this paper is to present an original method to take into account the coupling between rotation and translation stages in the robot's model in order to reduce its impact. The construction, identification and experiments will be done on a robotic device designed for micro-assembly.

In section II, the robotic device used to test our approach is presented. Section III deals with the description of the geometrical model. Then, the calibration procedure of the robotic device is proposed in section IV. Finally performances and discussions on the method are summarized in section V. 


\section{Micro-Assembly Robotic Device}

\section{A. Presentation of the device}

Our current robotic micro-assembly device (see in figure 1) is able to realize micro-assembled parts whose size is from $100 \mu \mathrm{m}$ to few micrometers. Tridimensional micro-assembly are currently done in teleoperation[9] and some automatic pick-and-place operations which use only translation stages are currently available.

Actuation is divided into two groups which has 3 degrees of freedom (DOF). The first one allows displacement of the substrate, where microparts are placed. Two linear and one rotation DOFs are available in the horizontal plane. The second group is the a 'robotic arm', composed of one linear DOF along the vertical axis and two rotation DOFs to ensure pitch and roll rotations of the microgripper. Moreover, the microgripper is based on a $4 \mathrm{DOFs}$ piezoelectric actuator[10] with silicon finger tips[11]. This kind of gripper is able to produce motions with great resolution (few tenth of nanometers).

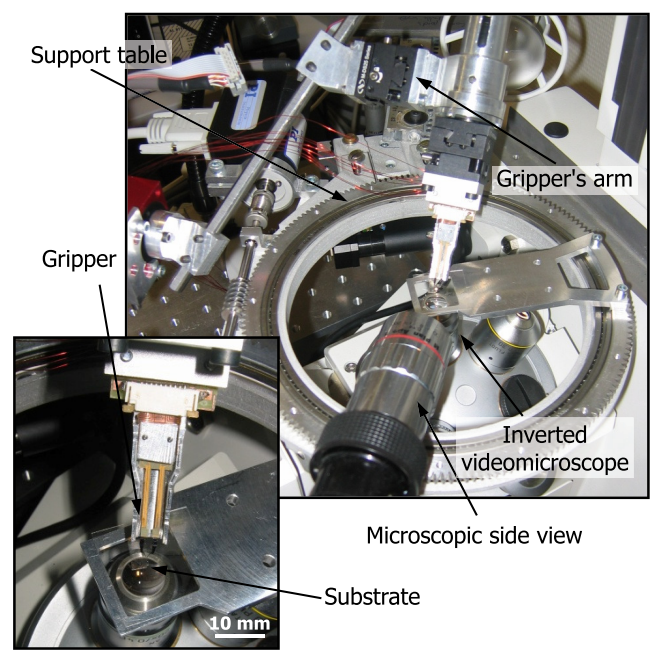

Fig. 1. Microassembly robotic device

Microscopical vision is provided by two videomicroscopes. As the volume above the micromanipulation plane is dedicated to microgripper movement, an inverted microscope LEICA DM-IRBE is used. It also allows micro-assembly in liquid medium, whose interest is synthesize in [12]. A second view for teleoperated operations is given by a side videomicroscope.

Based on handling strategies proposed in [9], many assemblies of microscopic mechanical parts have been made with the device in teleoperation (see in figure 2). Unfortunately, without geometric models, rotations are difficult to control for the human operator. The operator must be helped by robotic geometric models and adapted controls.

\section{B. Planar robotic device}

Present article framework focuses on a 3 DOFs subsystem of the whole robotic structure to illustrate our original method to reduce coupling between rotation and translation.
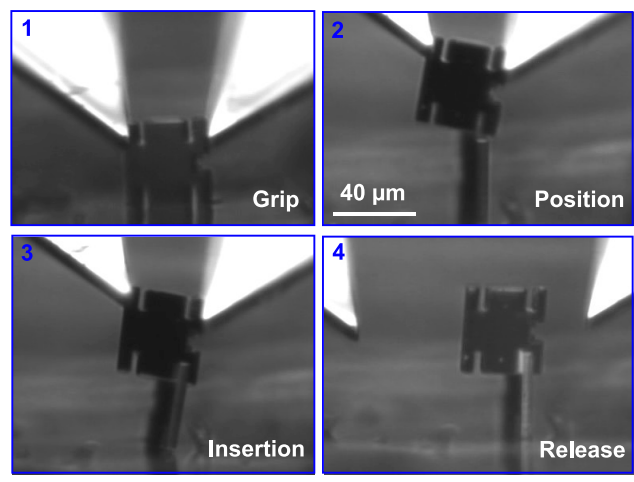

Fig. 2. Typical teleoperated assembly.

We consider the planar robotic device where the microobjects are placed (see in figure 3 ).

To move the substrate with great accuracy in the horizontal plane, two linear stages (Physick Instrumente, M111.1DG) are used. These stages have the following properties: linearity less than $2 \mu \mathrm{m}$ on all range, uni-directionnal repeatability about $100 \mathrm{~nm}$ and and bi-directional repeatability less than $2 \mu \mathrm{m}$. The design of the rotation stage is highly constrained by the size of the microscope. The rotation is realized with standard ball bearing and guiding has thus a micrometric concentricity around $65 \mu \mathrm{m}$. This value is usually sufficient at large scale but it is huge compared to the repeatability expected in micro-assembly. This example clearly illustrates the coupling between linear and angular stages which could highly perturb the performances of linear positions. Therefore, we propose to include these defaults in a geometrical model in order to improve the performance of the robotic structure.

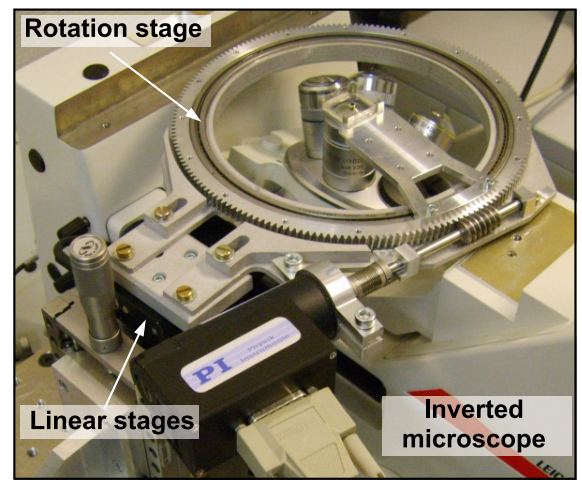

Fig. 3. 3DOF Planar Robotic System Studied

\section{Modeling OF The 3 DOF PlanAR ROBOtiC DEVICE}

Currently, the robot used in micromanipulation are build by assembly of commercial micropositioning stages. To build a precise geometric model of this kind of robots, two types of defaults must be considered:

- First, some defaults are intrinsic to the positioning stages: linearity or concentricity of the guidings, unidirectional and bidirectional repeatability, and accuracy 
are often limited by the mechanical design of the stages (linear or circular guide, reduction gear backlash, friction, etc.).

- Secondly, some defaults come from the assembly of the stages: coplanarity of each stage and perpendicularity of both linear stages.

Some of these defaults can be corrected by using a precise geometrical model of the robot and without using close-loop control. In fact, when repeatability is 'good' but accuracy is 'poor', a model of the robotic structure can be used to improve accuracy in open-loop control as shown in figure 4 .

For automatic assembly of micro-objects whose typical size is about $10 \mu \mathrm{m}$, an accuracy close to $200 \mathrm{~nm}$ is required which cannot be reached only with a model of the robotic structure. In that case, vision feedback has to be considered. In other hand, teleoperated control of assembly can be realized with a robotic structure which has a larger accuracy (typically up to 5 micrometers). In both cases, a geometric model is required during rotation to allow micro-objects reorientation with minor disturbance on the linear positions.

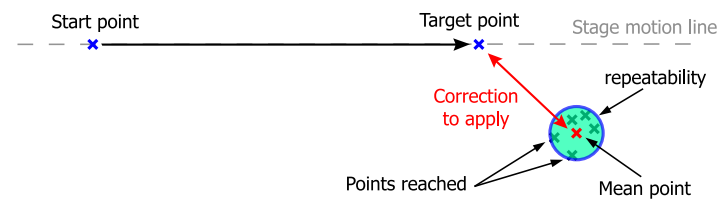

Fig. 4. Linear motion correction with great repeatability and poor accuracy

\section{A. Scope of the modeling}

Some experimental tests have been carry out to identify the predominant defaults. Two major defaults have been identified and will be include in the model:(i) the perpendicularity default between linear stages (some degrees), (ii) the rotation stage's concentricity ( $65 \mu \mathrm{m}$ maximum disturbance on objects circular trajectory, see in figure 5).

In fact, we assume in our model that the position errors induced by the linearity's defaults and the repeatability's defaults of the linear stages are negligible. Both defaults considered are quite repeatable and can be corrected with a geometrical model. Concerning the concentricity, the default is quite repeatable for each stage complete turn, and disturbance range from one turn to another is evaluated to $1.65(+/-0.55) \mu \mathrm{m}$ (see in figure 6).

\section{B. Geometrical modeling}

Defaults measurements of the planar robotic structure can be done by computer vision using the inverted microscope [8], [13], [14]. The reference (or fixed) frame $R_{0}$ is thus defined as the image's frame.

The model presented here is used to link micro-object's frame to reference frame $R_{0}$ (see in figure 7). Three frames are thus defined:

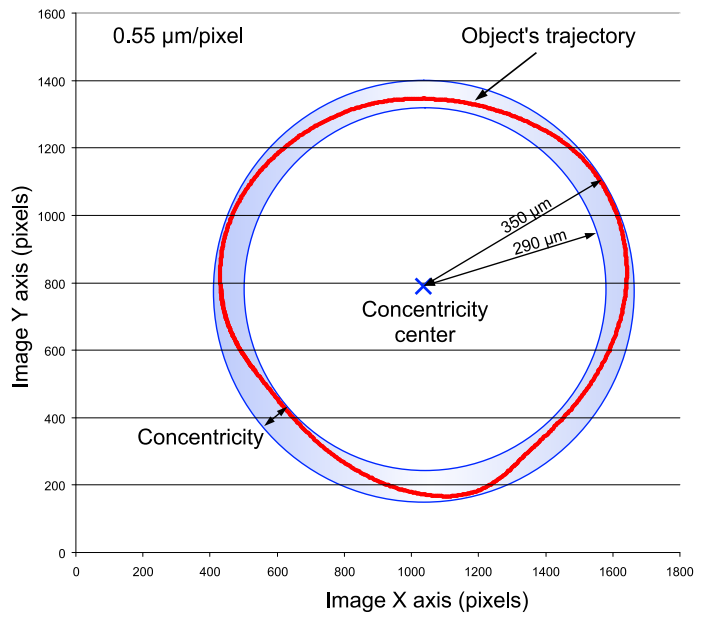

Fig. 5. Micro-object trajectory for $360^{\circ}$ on rotation stage.

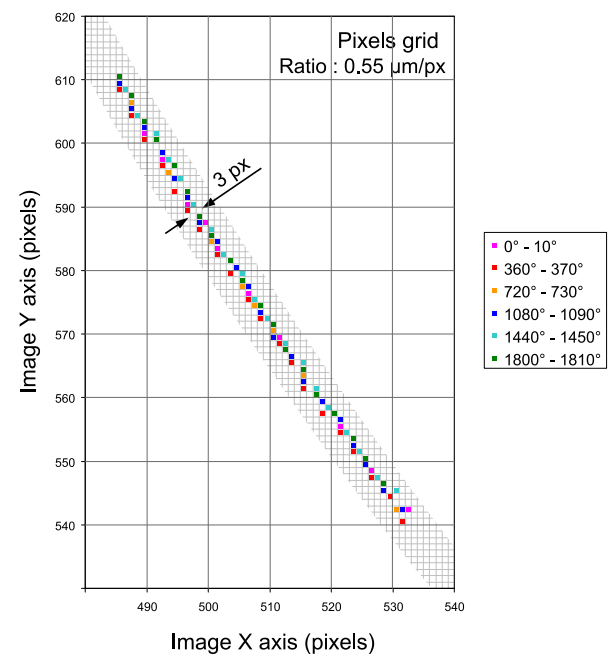

Fig. 6. Trajectory repeatability on $10^{\circ}$ range for 6 turns.

- $R_{1}^{\prime}$ is not orthogonal, both axis $x_{1}^{\prime}$ and $y_{1}^{\prime}$ is colinear to the direction of both linear stages. The distance between the center $O_{1}$ of $R_{1}^{\prime}$ is a linear function of the translation $c_{x}, c_{y}$ of both linear stages:

$$
\vec{O}_{0} O_{1}=c_{x} \cdot \vec{x}_{1}^{\prime}+c_{y} \cdot \vec{y}_{1}^{\prime}
$$

- $R_{1}$ is orthogonal, oriented like image frame and its center is $O_{1}$.

- $R_{2}$ is orthogonal, oriented from $R_{1}$ with the angular parameter $\theta$. This frame models the rotation stage, and $\mathrm{O}_{2}$ the center of the frame represents the center of rotation.

Finally, the center of gravity of the object is represented by the point $M$, fixed in $R_{2}$.

First, the modeling of the orientation defaults between the linear stages $R_{1}^{\prime}$ and the camera frame $R_{0}$ is defined by two angles: $\psi$ is the angle between both linear stages (close to $90^{\circ}$ ) and $\phi$ is the angle between image's horizontal axis $x_{0}$ and the first linear stage axis $x_{1}^{\prime}$. The translation $\left(c_{x}^{\prime}, c_{y}^{\prime}\right)$ of 


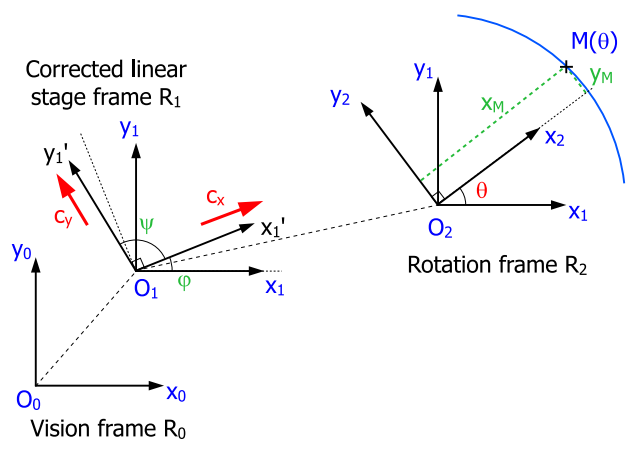

Fig. 7. Geometric model frames

$O_{1}$ in the camera frame $R_{0}$ can be thus defined in function of the real translations of the linear stages $\left(c_{x}, c_{y}\right)$ :

$$
\begin{aligned}
\overrightarrow{O_{0} O_{1}} & =c_{x}^{\prime} \cdot \vec{x}_{1}+c_{y}^{\prime} \cdot \vec{y}_{1} \\
\text { where } \quad c_{x}^{\prime} & =\left(c_{x} \cdot \cos (\phi)+c_{y} \cdot \cos (\phi+\psi)\right) \\
\text { and } \quad c_{y}^{\prime} & =\left(c_{x} \cdot \sin (\phi)+c_{y} \cdot \sin (\phi+\psi)\right)
\end{aligned}
$$

This simple model is able to define the trajectory of the point $O_{1}$ in the image in function of the displacement of both linear stages.

Without concentricity defaults, rotation center $\mathrm{O}_{2}$ of the stage stays fixed in $R_{1}$. In our case, we take into consideration the concentricity defaults, and we thus consider that distance $O_{1} O_{2}$ changes during a rotation. As, the trajectory is quite repeatable for each turn, $O_{1} O_{2}$ coordinates in $R_{1}$ can be considered only dependent of $\theta$ :

$$
\vec{O}_{1} O_{2}=X(\theta) \cdot \vec{x}_{1}+Y(\theta) \cdot \vec{y}_{1}
$$

Finally, the model is able to define the position of a point $M$ in function of fixed parameters $\left(\phi, \psi, x_{M}\right.$ and $\left.y_{M}\right)$, robotic linear and angular commands $\left(c_{x}, c_{y}\right.$ and $\theta$, ) and rotation defaults $X(\theta)$ and $Y(\theta)$ :

$$
\begin{aligned}
\overrightarrow{O_{0} M} & =\overrightarrow{O_{0} O_{1}}+\overrightarrow{O_{1} O_{2}}+\overrightarrow{O_{2} M} \\
& =c_{x} \cdot \vec{x}_{1}^{\prime}+c_{y} \cdot \vec{y}_{1}^{\prime}+X(\theta) \cdot \vec{x}_{1}+ \\
Y(\theta) \cdot \overrightarrow{y_{1}}+x_{M} & \overrightarrow{x_{2}}+y_{M} \overrightarrow{y_{2}} \\
\overrightarrow{O_{0} M} / R_{0} & =\left(\begin{array}{l}
c_{x} \cdot \cos (\phi)+c_{y} \cdot \cos (\phi+\psi) \\
c_{x} \cdot \sin (\phi)+c_{y} \cdot \sin (\phi+\psi)
\end{array}\right)+\left(\begin{array}{l}
X(\theta) \\
Y(\theta)
\end{array}\right) \\
& +\left(\begin{array}{cc}
\cos (\theta) & -\sin (\theta) \\
\sin (\theta) & \cos (\theta)
\end{array}\right) \cdot\left(\begin{array}{l}
x_{M} \\
y_{M}
\end{array}\right)
\end{aligned}
$$

This model can be used to improve open-loop control for position and orientation of a micro-object. The model parameters (orientation and rotation defaults) have to be measured during the robot calibration process.

\section{PARAMETERS IDENTIFICATION}

\section{A. Experimental setup}

Computer vision has been used to measure the position and orientation of multiple objects on the substrate. The vision sensor is a CCD sensor of $2044 \times 1500$ pixels, with a $10 \mathrm{x}$ optical objective, a resolution is $0.55 \mu \mathrm{m} / \mathrm{px}$ and the field of view is thus $1124 \times 825 \mu \mathrm{m}^{2}$. For calibration, two $40 \mu \mathrm{m}$ square shaped micro-objects are manually set on a transparent substrate. The substrate is placed manually close to the rotation stage center because rotation motion on several turns must not move micro-objects out of the field of view.

\section{B. Linear stages defaults}

Parameters $\phi$ and $\psi$, which defined the linear stages orientation in the image frame, could be measured by computation of the object trajectory during two translation along the linear stages directions $\left(\vec{x}_{1}^{\prime}, \vec{y}_{1}^{\prime}\right)$. Both angles are given with an accuracy of $0.05^{\circ}$. Angle $\psi$, which represents the orthogonality default of both stages, was evaluated to $92^{\circ}$. This kind of experimental measurement could give more useful data like stage accuracy, repeatability and linearity on the image range.

\section{Rotation stage defaults}

Defaults of the rotation axis represented by $X(\theta)$ and $Y(\theta)$ must be identify too. The position of both objects' trajectory is thus measured on several turns, with a sampling of 720 values per turn. Then two different methods have been tested to identify parameters $X(\theta)$ and $Y(\theta)$ of the model.

The first method is based on the measurement of the velocity of the point $\mathrm{M}$ which could be calculated from equation (4):

$$
\begin{array}{r}
V\left(\vec{M} / R_{0}\right)=\dot{c}_{x} \cdot \vec{x}_{1}^{\prime}+\dot{c}_{y} \cdot \vec{y}_{1}^{\prime}+\dot{X}(\theta) \cdot \vec{x}_{1}+ \\
\dot{Y}(\theta) \cdot \vec{y}_{1}+\dot{\theta} \cdot x_{M} \overrightarrow{y_{2}}-\dot{\theta} \cdot y_{M} \overrightarrow{x_{2}}
\end{array}
$$

During the identification of rotation defaults, the linear stages is not used:

$$
\dot{c}_{x}=\dot{c}_{y}=0 .
$$

The first hypothesis consists in neglecting the velocity induced by the rotation default $(\dot{X}, \dot{Y})$ compared to the velocity induced by the rotation itself $(\dot{\theta})$. In that case, $\mathrm{O}_{2}$ is considered as the Instantaneous Center of Rotation (ICR). Vector $\overrightarrow{O_{2} M}$ and the velocity of the point $M$ is thus always orthogonal and the trajectories of two points are sufficient to determine the position of $O_{2}$ for each angular position $\theta$ (see left part of figure 8).

As the trajectory is given by a discrete list of points, the noise on the measurement of the velocity has been reduced by using an interpolation of the experimental trajectory. Finally, an ICR identifying algorithm was applied on the whole trajectories of two points and ICR coordinates were stored. Nevertheless, these coordinates did not fit with known default: ICR motion range was close to a $200 \mu \mathrm{m}$ diameter circle whereas concentricity default is bounded by two circles distant from $65 \mu \mathrm{m}$. Moreover, implemented model did not compensate correctly concentricity default of the stage. As a conclusion, this first hypothesis is too large to build a right model. Nevertheless this approach will be used to initialize the second one presented in the following. 

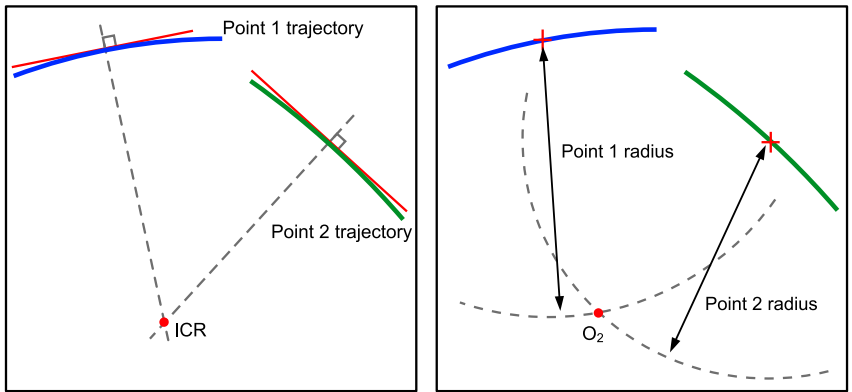

Fig. 8. Both ways to find out the rotation defaults $(X(\theta), Y(\theta))$ for one $\theta$.

A second method to measure rotation default is based on the fact that parameters $x_{M}$ and $y_{M}$ are constant for all $\theta$. Then, distance $\sqrt{x_{M}^{2}+y_{M}^{2}}$ between $O_{2}$ and $M$ is constant too. We now consider the trajectory of two objects $M_{1}$ and $M_{2}$. If constant distance $O_{2} M_{1}$ and $O_{2} M_{2}$ is known for an initial $\theta$, all others $\mathrm{O}_{2}$ coordinates are available by intersection of two trajectories points circles whose radius are $O_{2} M_{1}$ and $O_{2} M_{2}$ (see right part of figure 8). This algorithm is able to provide the position $(X(\theta), Y(\theta))$ of $O_{2}$ without hypothesis but it requires the initialization of circle radius $\left(\mathrm{O}_{2} M_{1}, \mathrm{O}_{2} M_{2}\right)$. Then the first identifying method is used to make this initialization, with arithmetic mean of all measured distance between points and the point $\mathrm{O}_{2}$ identified by the first method (see in figure 9). Finally, a new identification of parameters $(X(\theta), Y(\theta))$ has been obtained. These parameters obtained with the second method corresponds with the expected range (see in figure 10).

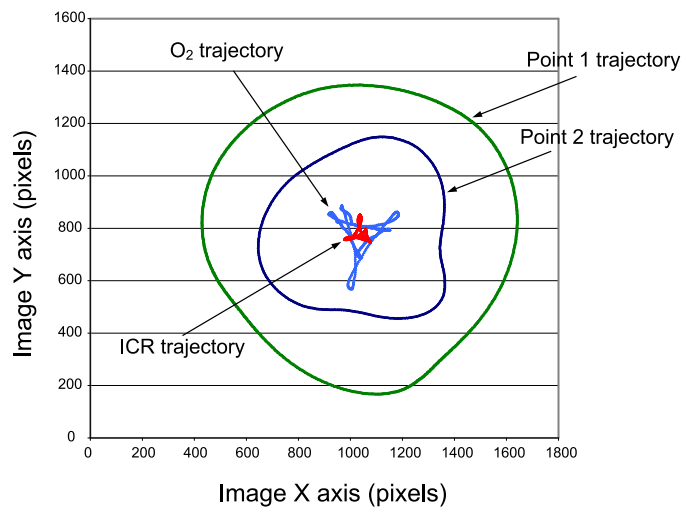

Fig. 9. Identification of $\mathrm{O}_{2}$ trajectory from the gravity center trajectory of two micro-objects.

\section{PERFormances}

\section{A. Validation}

To validate the identification procedure of the parameters $(X(\theta), Y(\theta))$, the identification have been made with three trajectories. As the identification can be done only two curves, three curves give three possible couples and then three kind of parameters $(X(\theta), Y(\theta))$. It could be interesting to note than the three curves have exactly the same shape and

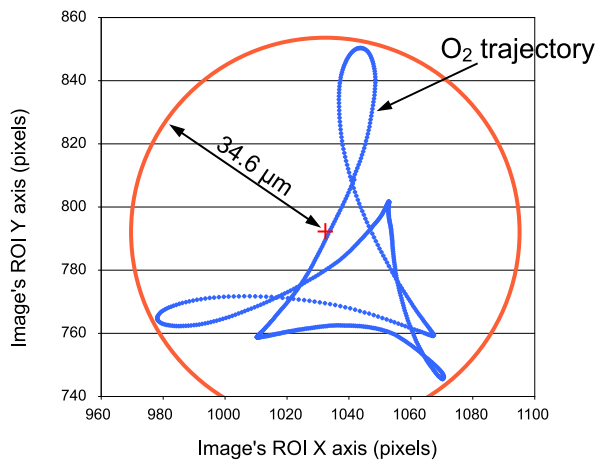

Fig. 10. $\mathrm{O}_{2}$ trajectory shape corresponding to concentricity default value.

orientation, but are translated of up to $3 \mu \mathrm{m}$ from each others. Radius initialization and precision of the vision measurement seems to be the two major explanations of these translation. To reduce the impact of this default, $(X(\theta), Y(\theta))$ values have been averaged. Finally, the whole geometrical model has been build in MatLab, and correction has been tested on a fourth trajectory which was not used for identification. As a result, corrected trajectory of this object have a better circularity than the non-corrected one, (see in figure 11).

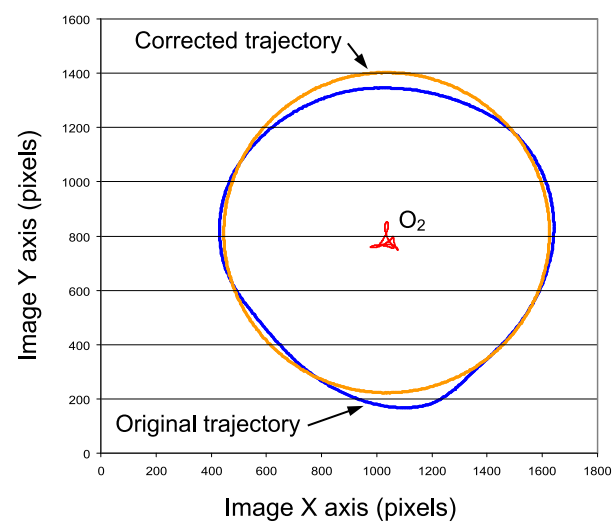

Fig. 11. Corrected trajectory of fourth point.

Model was tested in simulation to made a rotation of the fourth object around its gravity center (see in figure 12). Results clearly shows than the model improve rotation behavior, and positioning default was reducted from $65 \mu \mathrm{m}$ to $4.4 \mu \mathrm{m}$ for a complete turn. For $60^{\circ}$, the default is estimated to $1 \mu \mathrm{m}$ (eg. 2 pixels) in both direction. Then for small change of orientation, positioning default was very small, and narrowly detectable by our vision sensor.

\section{B. Discussion}

The proposed model is able to improve robotic system repeatability up to $93 \%$ in linear position for a complete turn. The accuracy is thus highly improved by the model. But this model can be refined to reach vision sensors limit and repeat current behaviour for $60^{\circ}$ re-orientation to full turn behaviour. We propose to use more test points, to reduce in terpolation defaults and improve rotation defaults coordinates 


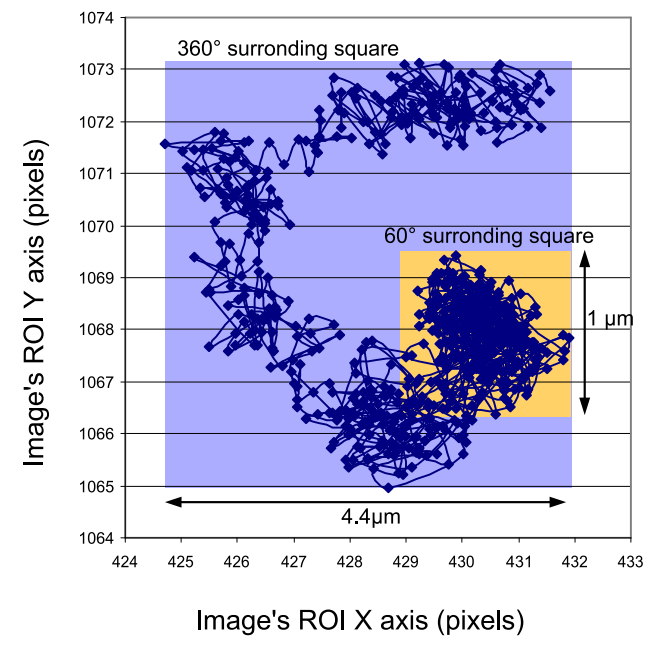

Fig. 12. Gravity center trajectory of fourth point for rotation around it.

averaging. Vision patterns, already used in vision calibration and made in silicon in our laboratory (see in figure 13), will be used to replace micro-objects. With 20 squares of $20 \mu \mathrm{m}$ side, this pattern will offer 210 identification couples instead of 3 in this study.

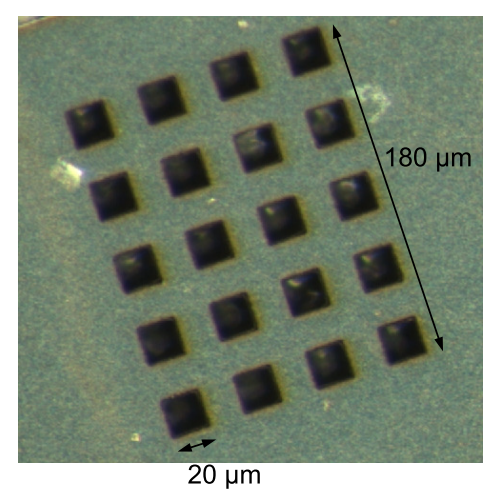

Fig. 13. Visual calibration pattern.

The time for calibration is although a criteria of performance. In our case, only micro-object tracking is used to find their gravity center. With a camera frame rate close to $25 \mathrm{fps}$, and a desired data sampling of $0.5^{\circ}$, one turn calibration time is evaluated to 29 seconds. Identification algorithm is very quick and don't exceed few seconds. Finally, in less than 1 minute, the full calibration of the presented rotational device is possible and correction is then applied quickly.

\section{CONCLUSION AND PERSPECTIVES}

This article proposes an innovative way to improve microscopic behavior of macroscopic rotation stage commonly used in robotic micro-assembly. The proposed geometrical model of a planar robot (2DOF in translation and $1 \mathrm{DOF}$ in rotation) take into account the angular default between linear stages and the concentricity defaults of the rotation stage. Both defaults are predominant in the microscale. The calibration proposed requires the identification of specific parameters which characterize the concentricity defaults during a rotation. Two identification procedures have been proposed and discussed and the model has been tested. The improvement on the repeatability of the linear position reaches $93 \%$ for a complete turn. This model allows microobjects dynamically re-orientation with minor linear motions.

In future work, the presented model will be tested on an micro-object automated pick-and-place to validate the geometrical model in an application field. Calibration will be improved by using more points for rotation defaults' identification. To improve the model, integration of XY stages linearity defaults, backlash and coplanarity errors will be studied.

\section{REFERENCES}

[1] B. López-Walle, M. Gauthier, and N. Chaillet. A submerged freeze microgripper for micromanipulations. In International Conference on Robotics and Automation (ICRA), Roma, Italy, April 2007.

[2] M. Gauthier, E. Gibeau, and D. Hériban. Submerged robotic micromanipulation and dielectrophoretic micro-object release. In proc. of the IEEE ICARCV 2006 conference, Singapour, dec. 2006.

[3] B. Tamadazte, S. Dembélé, and N. Le Fort-Piat. Multiscale calibration of a photon video microscope. Submitted to IEEE International Conference on Intelligent Robots and Systems (IROS), 2008.

[4] F. Beyeler, A. P. Neild, S. Obert, D. J. Bell, Y. Sun, J. Dual, and B. J. Nelson. Monolithically fabricated micro-gripper with integrated force sensor for manipulating micro-objects and biological cells aligned in an ultrasonic field. IEEE/ASME Journal of Microelectromechanical Systems (JMEMS), 16(1):7-15, 2007.

[5] Akihiro Matsumoto and Kunio Yoshida. Design of desktop microassembly machine and its industrial applications. In Worskshop robotic microassembly - IEEE IROS 2007, San Diego, oct. 2007.

[6] John Wason, William Gressick, John T. Wen Jason Gorman, and Nick Dagalakis. Multi-probe micro-assembly. In Proceedings of the $3 \mathrm{rd}$ Annual IEEE Conference on Automation Science and Engineering, pages 63-68, Scottsdale, AZ, USA, Sept 22-25 2007.

[7] S. Venugopal, L-C Hsu, S Malalur-Nagaraja-Rao, B.P. Wang, M. Chiago, and J-C. Chiao. Design and modeling of a high accuracy, three degree of freedom mems manipulator. In SPIE 2005 Microelectronics, MEMS, and Nanotechnology Symposium, DEvice And Process Technologies For Microelectonics, MEMS and Photonics Conference, Brisbane, Australia, december 2005

[8] Tatsuo ARAI, Akihiro SUZUKI, Yosuke KATO, Yasushi MAE, Kenji INOUE, and Tamio TANIKAWA. Automated calibration for micro hand using visual infomation. In In Proc. of the 2002 IEEE/RSJ Int. Conference on Intelligen Robots and Systems, pages 293-98, Lausanne, Switzerland, October 2002.

[9] D. Heriban and M. Gauthier. Robotic micro-assembly of microparts. In Submitted in IEEE/RSJ IROS Conference, Nice, France, Feb. 2008.

[10] J. Agnus, P. Nectoux, and N. Chaillet. Overview of microgrippers and design of a micromanipulation station based on a mmoc microgripper. In Proc. of IEEE CIRA, Finland, 2005.

[11] David Heriban, Joël Agnus, Jean-René Coudevylle, Michaël Gauthier, and Nicolas Chaillet. Design of silicon finger tips for a moc (microrobot on chip) microgripper. In Proc. of the Int. Workshop on Topica Meeting on Microfactories (TMMF05), Tsukuba, Japan, October 2005.

[12] M. Gauthier, S. Régnier, P. Rougeot, and N. Chaillet. Forces analysis for micromanipulations in dry and liquid media. Journal of Micromechatronics, 3(3-4):389-413, Sept. 2006.

[13] Guangyong Li and Ning Xi. Calibration of a micromanipulation system. In Proc. of the 2002 IEEE/RSJ Int. Conference on Intelligen Robots and Systems, pages 1743-47, Lausanne, Switzerland, October 2002.

[14] Aaron M. Hoover and Ronald S. Fearing. Rapidly prototyped orthotweezers for automated microassembly. In Proc. of the IEEE ICRA 2007 Conf., pages 812-19, Roma, Italy, EU, April 2007. 